\title{
An LED SAHP-based Planar Projection PTCDV-hop Location Algorithm
}

\author{
Yuexia Zhang ${ }^{1,2 *}$, Hang Chen ${ }^{1,2}$, Jiacheng Jin $^{1,2}$ \\ ${ }^{1}$ School of Information and Communication Engineering Institute, Beijing Information Science \& Technology \\ University, Beijing, 100101, China \\ 2 Beijing Key Laboratory of High Dynamic Navigation Technology, Beijing Information Science \& Technology \\ University, Beijing, 100101, China \\ [e-mail: zhangyuexia@bistu.edu.cn] \\ *Corresponding author: Yuexia Zhang
}

Received September 27, 2018; revised February 8, 2019; accepted March 18, 2019;

published September 30, 2019

\begin{abstract}
This paper proposes a planar projection DV-hop location algorithm (PTCDV-hop) based on the LED semi-angle at half power (SAHP, which accounts for LED SAHP characteristics in visible light communication (VLC)) and uses the DV-hop algorithm for range-free localization. Distances between source nodes and nodes positioned in three-dimensional indoor space are projected onto a two-dimensional plane to reduce complexity. Circles are structured by assigning source nodes (projected onto the horizontal plane of the assigned nodes) to be centers and the projection distances as radii. The proposed PTCDV-hop algorithm then determines the position of node location coordinates using the trilateralweighted-centroid algorithm. Simulation results show localization errors of the proposed algorithm are on the order of magnitude of a millimeter when three sources are used. The PTCDV-hop algorithm has higher positioning accuracy and stronger dominance than the traditional DV-hop algorithm.
\end{abstract}

Keywords: DV-hop algorithm; indoor positioning; semi-angle at half power (SAHP); visible light communication(VLC)

This research was supported by the National Natural Science Foundation of China(No.51334003,No.61473039), the Introduction and Training Program of high level talents in Beijing municipal colleges and Universities (No.CIT\&TCD201504058), and the Beijing Information Science and Technology University Graduate Education Quality Engineering Project (No.5121724107). 


\section{Introduction}

Public demand for location services has increased with developments in communication network technology [1]-[2]. As statistics indicate people spend 70\% of their time in family, office, and other indoor environments, demands for indoor positioning are growing in urgency. Global positioning system (GPS) currently plays an important role in the field of positioning; however, it is difficult to receive GPS satellite signals in indoor environments. Visible light communication (VLC) has become a new type of green communication technology as communication networks have developed, using white light LEDs to conduct high-frequency light and dark scintillation signals and actualize high-speed data transmission [3]-[5]. VLC technology may be conveniently applied and has a high positioning precision, low cost, and low power consumption. Furthermore, it is well-suited to indoor environments. In 2011, China announced an elimination roadmap for incandescent lamps, allowing the LED to become the next generation of lighting technology. Researchers have thus worked hard to put forward numerous techniques for achieving system performance with advantages such as low cost and high positioning precision [6].

Location algorithms can categorized as either range-based or range-free. Significant research exists regarding VLC range-based algorithms.For example, Hyun-Seung Kim calculated distance information by separating received signal strengths from different sources, achieving an indoor positioning accuracy in the centimeter range [7]; Do T H et al. proposed an enhanced time difference of arrival (TDOA) algorithm for indoor positioning without LED-ID by using visible light radiated from LEDs to locate receiver positions with high estimation accuracy $(3.6 \mathrm{~cm})$ in computer simulation [8]; and Nan W et al. proposed the MLED-RSS positioning algorithm based on received signal strength (RSS), utilizing multiple LED transmitters for indoor positioning, effectively solving the block or shadow effect existing in visible indoor locations, and producing simulation results demonstrating the high localization accuracy of the MLED-RSS positioning algorithm [9]. Xudong W et al. proposed an improved TDOA localization algorithm with centroid weighting, achieving an average positioning error $3 \mathrm{~cm}$ below the SNR of 2dB [10]. In [11], Xueli Zhang et al. analyzed the Cramer-Rao lower bound (CRLB) and indoor noise interference of an RSS algorithm to achieve centimeter-level positioning accuracy. A new alpha-stable distributionbased relative positioning model using time of arrival (TOA) measurements to obtain the relative positional relationship between two locators was proposed in [12]. In [13], a statistical ranging error model was proposed for non-line of sight (NLOS) environments based on empirical ranging data, substantially improving positioning accuracy and achieving seat-level accuracy for $99 \%$ of tested nodes. Finally, in [14], 3D positioning was estimated using RSS and angle-of-arrival (AOA)-based hybrid positioning algorithms, achieving 7.3 and $67.2 \mathrm{~cm}$ mean positioning errors. Above all, positioning errors have been reduced in range-based methods.

Range-free location algorithms such as the DV-hop, characterized by their low cost, low power consumption, strong anti-noise abilities, sand simple hardware, have received much attention from researchers. For example, Li et al.[15] proposed an efficient and secure rangefree localization scheme based on outlier elimination and the vector refinement process. An outlier elimination method was introduced into the localization process by filtering 
inaccurate beacon nodes in the presence of malicious beacon nodes, and the results demonstrated significant improvements in localization accuracy relative to the traditional DV-hop algorithm. In [16], an approximate formula for the optimal RSS threshold value, showing the minimum error for a particular network to be localized, was derived as a function of the total number of nodes and the network size, allowing the error to be minimized and maintain applicability to both small- and large-scale networks. Their results confirmed that utilizing the proposed RSS threshold-based connectivity configuration significantly improved the localization accuracy of DV-hop in a practical environment without any extra hardware. Little research, however, has investigated the DV-hop algorithm in the VLC field, and existing studies failed to consider positioning accuracy variations in different LED receiving angles.

This paper proposes a projection DV-hop location algorithm (PTCDV-hop) that uses the DV-hop algorithm in range-free localization and is based on the LED semi-angle at half power (SAHP), accounting for LED SAHP characteristics in VLC. Distances between source nodes and nodes to be positioned in three-dimensional indoor spaces are projected onto a two-dimensional plane to reduce complexity. Circles are structured by assigning the source nodes (projected onto the horizontal plane of the positioned node) as centers and the projection distances as radii. The proposed PTCDV-hop algorithm then determines the location coordinates of the node to be positioned using a trilateral-weighted-centroid algorithm. Simulation results demonstrated the localization error of the PTCDV-hop algorithm is on the order of magnitude of a millimeter when three sources are used. The PTCDV-hop algorithm had higher positioning accuracy and stronger dominance than the traditional DV-hop algorithm.

The remainder of this paper is organized as follows: the system model is presented in Section II, the PTCDV-Hop algorithm is introduced in Section III, Section IV analyzes the simulation results, and Section Vpresents concluding remarks.

\section{The system model}

\subsection{VLC System Model}

The VLC system channel transmission model is shown in Fig. 1 [17]. The source $S$ sends out the modulated optical signal $X(t)$, and the destination $D$ uses the photodiode to receive the signal $Y(t)$.

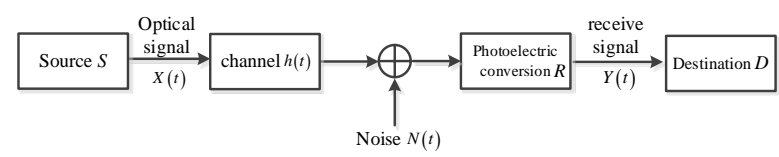

Fig. 1. VLC system channel transmission model

Essentially, the operating principles of a VLC system resemble those of a line-of-sight wireless communication system. Instead of transmitting and receiving RF signals, VLC systems transmit and receive optical signals in the visible wavelength region. There are many modulation methods available in wireless optical communication, including on-off keying (OOK), pulse position modulation (PPM), differential pulse position modulation (DPPM) and digital pulse interval modulation (DPIM). 
Intensity modulation/direct detection (IM/DD) systems, primarily of OOK modulation modes, are widely used in wireless optical communication [18]-[19]. The research in [20] proposes modified pulse position modulation (MPPMD) and variable pulse position modulation (VPPM) based on PPM, which minimizes complexity at the receiver. The MPPMD demonstrated the same bit error rate (BER) performance as traditional coherent demodulators. Additionally, the VPPM receiver was dramatically reduced when the multiplication stage and mask generator were removed. Reverse pulse position modulation (RPPM) was proposed in [21], providing a higher average transmit power and a better SNR than alternative modulation methods. The RPPM symbol waveform is shown in Fig. 2, demonstrating that signals are launched in timing gaps provided by the transmitter, preventing interference. Signals are composed of source and data location information. DPPM modulation can both solve the interference problem and improve the frequency utilization [22]-[24]. In [25], it was found that the spectral characteristics of DPIM could be achieved by employing a relatively narrow bandwidth data transmission. The construction of a narrow band-pass amplifier circuit requires few components and establishes a unidirectional VLC system. Experimental results demonstrated that the VLC system could actualize unidirectional data transmission with a transmission rate of approximately $45.8 \mathrm{Kbit} / \mathrm{s}$ within $5 \mathrm{~m}$ direct sight distance.

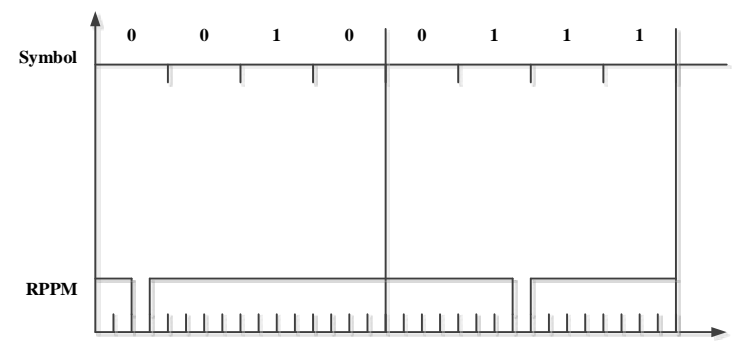

Fig. 2. Modulation symbol waveform of RPPM

The average light source transmission power of portable mobile communication devices should be as low as possible while maintaining an accurate BER due to human eye safety requirements and power restrictions. Power consumption, however, is less important in visible light communication than in mobile devices. Higher average power can reduce the number of LEDs used to meet indoor lighting brightness requirements. This paper applied the RPPM modulation method to a PTCDV-Hop algorithm positioning system. The VLC channel transmission process can be expressed as:

$$
Y(t)=R X(t) \cdot h(t)+N(t)
$$

where $R$ denotes the photoelectric sensor conversion efficiency, $N(t)$ is the white Gaussian noise, and $h(t)$ represents the channel impulse response. A VLC system channel is expressed as:

$$
\begin{gathered}
h(t)=\frac{A_{r}(m+1)}{2 \pi d^{2}} \cos ^{m}(\varphi) T_{s}(\psi) g(\psi) \cos (\psi) \delta\left(t-\frac{d}{c}\right) \\
0 \leq \psi \leq F O V
\end{gathered}
$$

where $d$ is the distance between the source and destination; $c=3 \times 10^{8} \mathrm{~m} / \mathrm{s} ; F O V$ denotes the source field angle; $A_{r}$ represents the receiving area of the photodetector; $\varphi$ and $\psi$ represent the radiation and receiving angles, respectively; $T_{s}(\psi)$ is the gain of the optical filter at the destination; $g(\psi)$ is the gain of the optical condenser; and $m$ is Lambert's radiation coefficient such that $m=-\ln 2 / \ln \left(\cos \varphi_{1 / 2}\right)$, where $\varphi_{1 / 2}$ represents the LED SAHP. 
Encapsulating the LED affects its luminous intensity. The LED radiation pattern in a VLC system is generally regarded to follow a Lambert's distribution. The LED luminous intensity and its emission angle obey the cosine model [26]:

$$
P(\phi)=P_{0} \cos \phi
$$

where $P_{0}$ is the central luminous intensity of the LED. Thus, the luminous intensity becomes half when $\varphi=\pi / 3$ (equal to $\varphi_{1 / 2}=\pi / 3$ ). As such, systems have better signal intensity, channel conditions, and communication effects when $\varphi<\varphi_{1 / 2}$, scilicet in the coverage of LED SAHP.

\subsection{VLC Indoor Positioning Model}

The VLC indoor positioning model shown in Fig. 3 represents a room with a $5 \mathrm{~m} \times 5 \mathrm{~m} \times \mathrm{Hm}$ positioning space, where $H$ is the known room height. Several white LED light sources $\left(S_{1}, S_{2}, \cdots, S_{i}\right)$ are arranged on the ceiling of the room at known coordinates $\left(x_{1}, y_{1}, z_{1}\right),\left(x_{2}, y_{2}, z_{2}\right) \cdots\left(x_{i}, y_{i}, z_{i}\right)$. PN represents the node to be located, which may be located at any position in the room with unknown coordinates $(x, y, z)$. The white LED light sources and the PN node can communicate with each other through the VLC system.In order to reduce energy consumption, the powers that be energy adaptive clustering hierarchy (LEACH) protocol [27] can be used for networking.

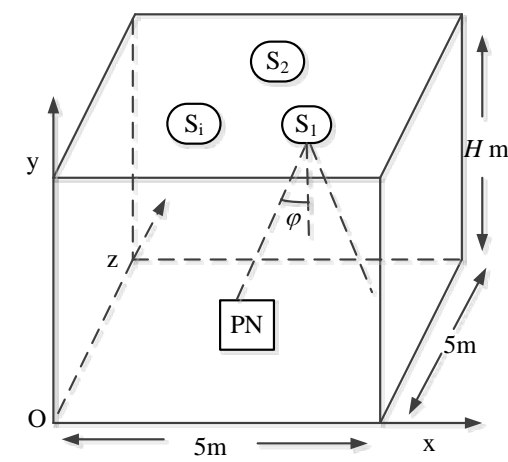

Fig. 3. VLC indoor positioning model

\section{PTCDV-Hop Algorithm}

\subsection{Traditional DV-hop Algorithm}

The traditional DV-Hop algorithm was proposed by Dragos Niculescu, among others [28][29], and relies on the routing hop number to identify a location without relying on sensor signal information. This reduces device costs and has a high adaptability. The location of the unknown node is estimated using preexisting information on the beacon node and the minimum hop number from the unknown node to the beacon node [30]-[32] . The steps of the traditional algorithm are as follows:

Step 1: The source node broadcasts its location information to the neighbor node, including the number of hops, and initializes the value to zero. The positioning node records the minimum number of hops required to reach each source node, and larger hop numbers are ignored. The hop value then adds one to the neighbor source node. 
Step 2: The source node calculates the average distance using a formula in accordance with the location and number of other source nodes it recorded. The distance between the node to be located and corresponding source node can then be determined.

Step 3: This distance can be calculated using the trilateral positioning coordinates once three distances between the locating and source nodes are obtained.

$$
\text { Hopsize }_{i}=\frac{\sum_{j \neq i} \sqrt{\left(x_{i}-x_{j}\right)^{2}+\left(y_{i}-y_{j}\right)^{2}}}{\sum_{j \neq i} \text { Hops }_{i}}
$$

where Hopsize $_{i}$ is the average distance of every hop; $\left(x_{i}, y_{i}\right)$ and $\left(x_{j}, y_{j}\right)$ represent the coordinates of source nodes $i$ and $j$, respectively; and Hops $s_{i}$ denotes the number of hops between nodes $i$ and $j$.

These steps are shown in Fig. 4.

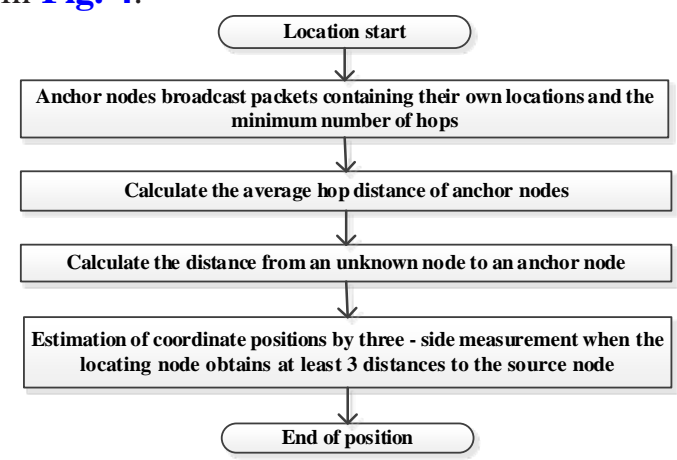

Fig. 4. Flowchart of traditional DV-hop algorithm

\subsection{Projection DV-hop VLC algorithm}

The PTCDV-hop VLC algorithm is shown in Fig. 5. The distance between the source node and the node to be positioned in a three-dimensional indoor space are mathematically projected onto a 2D plane to reduce the adverse effects of 3D space topology on indoor positioning results.

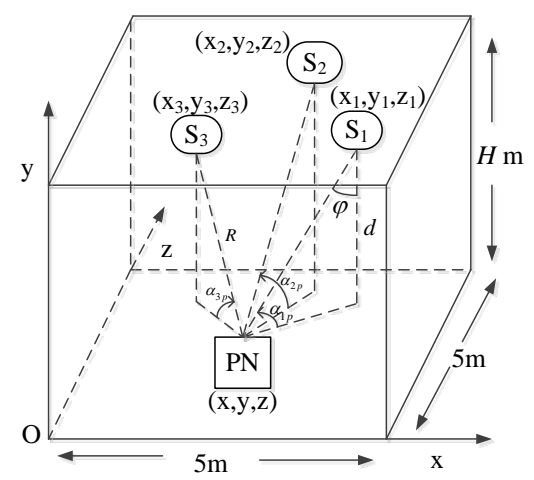

Fig. 5. Positioning model of the PTCDV-Hop VLC algorithm

Node PN is assumed to be within the source node SAHP coverage to ensure the best communication effect. The distance between the source node $S_{i}$ and node PN is $R$, calculated as follows:

$$
R=\sqrt{\left(x_{i}-x\right)^{2}+\left(y_{i}-y\right)^{2}+\left(z_{i}-z\right)^{2}}, i=1,2,3
$$


The minimum angle of incidence between nodes $S_{i}$ and PN is $\alpha_{i p}$, calculated as follows:

$$
\alpha_{i p}=\arcsin \frac{d}{R}, i=1,2,3
$$

where $d$ represents the vertical distance from node PN to the roof plane and $d=H-z$.

Because $\varphi_{1 / 2}=\pi / 3, \alpha_{i p \min }=\pi / 6$ and therefore the range of incidence angles $\alpha_{i p}$ is $[\pi / 6, \pi / 2]$. Due to the uncertainty of the location of the node PN, the actual incidence angles $\alpha_{i p}$ are distributed between ${ }^{\alpha_{i p \min }} \sim \pi / 2$, so the average value of $\left(\alpha_{i p \min }+\pi / 2\right) / 2$ is taken as an estimated angle of incidence. Subsequently, $\alpha_{i p}$ is calculated as follows:

$$
\alpha_{i p}=\left(\frac{\pi}{2}+\arcsin \frac{d}{R}\right) / 2, i=1,2,3
$$

The average hop distance of the VLC network is calculated using the known source node coordinates with Equation (8):

$$
\text { AHS }=\frac{\sum_{i} A H S_{i}}{i}, i=3
$$

The hop distance between $S_{i}$ and $\mathrm{PN}$ is then projected onto the horizontal plane according to Equations (5)-(8) as follows:

$$
L_{i p}=\left(A H S \cdot \cos \alpha_{i p}+\frac{d}{\tan \alpha_{i p}}\right) / 2, i=1,2,3
$$

\subsection{The Trilateral-weighted-centroid Algorithm}

Circles $O_{1}, O_{2}, O_{3}$ were structured by taking the three source nodes $S_{1}, S_{2}, S_{3}$ projected onto the horizontal plane of node PN as the centers and the $L_{1 p}, L_{2 p}, L_{3 p}$ from Equation (9) as the radii of the circle. Coordinates $(x, y, z)$ of $\mathrm{PN}$ are then calculated by the trilateration as

$$
\left\{\begin{array}{l}
L_{1 p}^{2}=\left(x_{1}-x\right)^{2}+\left(y_{1}-y\right)^{2} \\
L_{2 p}^{2}=\left(x_{2}-x\right)^{2}+\left(y_{2}-y\right)^{2} \\
L_{3 p}^{2}=\left(x_{3}-x\right)^{2}+\left(y_{3}-y\right)^{2}
\end{array}\right.
$$

Ideally, the three circles would intersect at one point in Equation (10); however, in reality they intersect each other to form an overlapping area because of measurement errors. The coordinates of node PN are therefore replaced by the centroid of the overlapping region, identified using the centroid algorithm. The intersection coordinates $\left(x_{O 12}, y_{O 12}, z_{O 12}\right)$ of circles $\mathrm{O}_{1}, \mathrm{O}_{2}$ are therefore calculated as

$$
\left\{\begin{array}{l}
L_{1 p}^{2} \geq\left(x_{1}-x_{O 12}\right)^{2}+\left(y_{1}-y_{O 12}\right)^{2} \\
L_{2 p}^{2}=\left(x_{2}-x_{O 12}\right)^{2}+\left(y_{2}-y_{O 12}\right)^{2} \\
L_{3 p}^{2}=\left(x_{3}-x_{O 12}\right)^{2}+\left(y_{3}-y_{O 12}\right)^{2}
\end{array}\right.
$$

Similarly, the intersection coordinates of $\left(x_{O 13}, y_{O 13}, z_{O 13}\right),\left(x_{O 23}, y_{O 23}, z_{O 23}\right)$, and the centroid of the overlap region are obtained using the weighted centroid. Coordinates $(x, y, z)$ of node PN are thus,

$$
\begin{aligned}
& x=\frac{x_{O 12}+x_{O 13}+x_{O 23}}{3} \\
& y=\frac{y_{O 12}+y_{O 13}+y_{O 23}}{3} \\
& z=\frac{z_{O 12}+z_{O 13}+z_{O 23}}{3}
\end{aligned}
$$




\subsection{Process of the PTCDV-Hop Algorithm}

Step 1: Node PN is randomly placed within the SAHP coverage of source nodes $S_{1}, S_{2}, S_{3}$ in the interior space of the roof. The coordinates of $S_{1}, S_{2}, S_{3}$ are known;

Step 2: Three different angles of incidence $\alpha_{1 p}, \alpha_{2 p}, \alpha_{3 p}$ are obtained from Equations (5)-(7) and the coordinates of $S_{1}, S_{2}, S_{3}$.

Step 3: The hop distances between $S_{1}, S_{2}, S_{3}$ and node PN projected onto the horizontal plane are $L_{1 p}, L_{2 p}, L_{3 p}$, and can be calculated using Equations (8)-(9) and $\alpha_{1 p}, \alpha_{2 p}, \alpha_{3 p}$ from Step 2;

Step 4: The coordinates of node PN are calculated using Equations (11)-(12) and $L_{1 p}, L_{2 p}, L_{3 p}$ from Step 3.

This positioning process is repeated from Steps 2-4 if node PN changes its position. Fig. 6 shows Steps 1-4 of the process of the PTCDV-Hop algorithm.

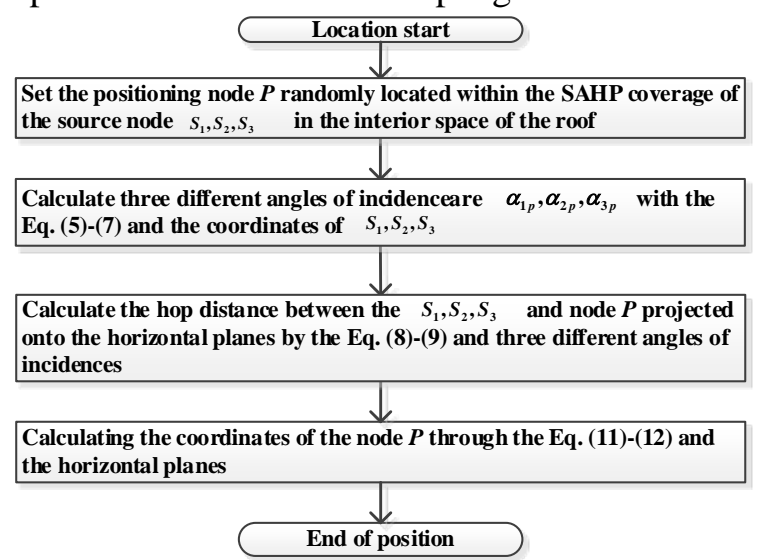

Fig. 6. PTCDV-Hop algorithm process

\section{Simulation Results And Analysis}

The simulation used parameters of $H=3$ and room model space $5 \mathbf{m} \times 5 \mathbf{m} \times 3 \mathbf{m}$. Fig. 7 presents a comparative diagram of the estimated and real locations of the node to be located $P N$ using three sources. The $\mathrm{X}, \mathrm{Y}$, and $\mathrm{Z}$ axes of the graph represent the length, width, and height of the room model, respectively. The circle represents the real position of $\mathrm{PN}$, and the triangle represents the estimated position calculated by the PTCDV-Hop algorithm.

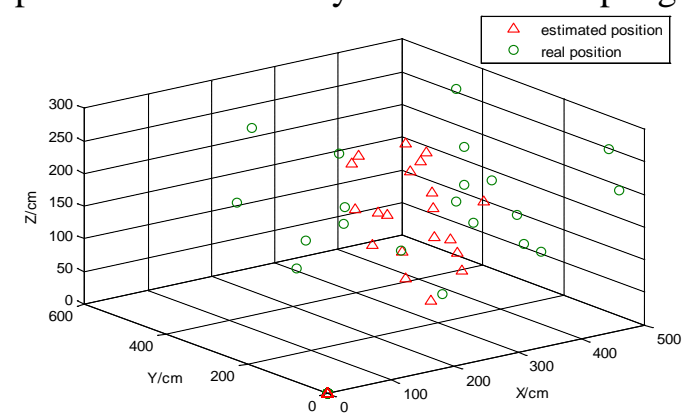

Fig. 7. Comparison of estimated and real PN positions 
It can be seen from Fig. 7 that the positioning accuracy is higher when the PN nodes are in the center of the room, and lower when the PN nodes are at the edge of room. This is because when the PN nodes are within the half power angular radiation range of the source node, the received signal strength is large;thus, the positioning accuracy is high. In contrast, when the PN nodes are at the edge of the room, outside the range of the half power angle radiation of the source node, the received signal strength is poor; thus, the positioning error is large. In order to improve the positioning accuracy of the PN nodes at the edge of the room, we conducted related studies. In one such study, we investigated the distribution of source nodes in the room, and proposed an IRT-N triangular array based on LED half power angle to improve the indoor LED half power angular radiation range, eliminate the "blind zone" of half power angle radiation, and reduce the located error [33]. At the same time, in a follow-up study, we are currently enhancing the algorithm proposed in this paper to improve the positioning accuracy of the PN nodes at the edge of the room.

Fig. 8 shows the root mean square error (RMSE) results of repeated PTCDV-Hop algorithm positioning for a random point using three sources in an indoor space. The abscissa indicates the positioning number and the ordinate represents the RMSE value. As shown, the RMSE value of multiple positioning results for the same point fluctuates slightly because of the influence of random errors, and the average RMSE value is approximately $<2.5 \mathrm{~cm}$. The simulation experiment showed that the PTCDV-Hop algorithm could achieve high positioning accuracy with errors $<2 \mathrm{~cm}$.

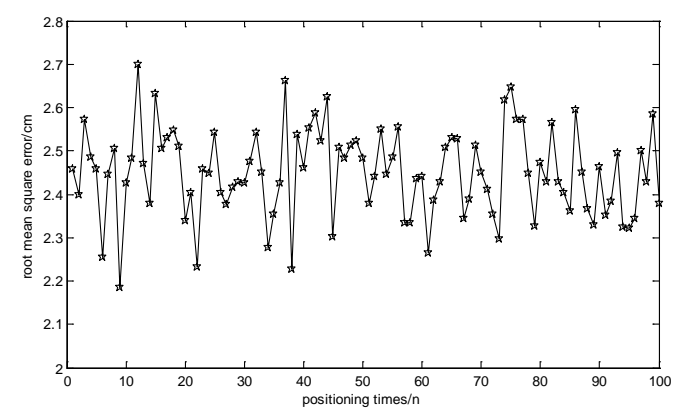

Fig. 8. RMSE values of PTCDV-Hop algorithm

To obtain an approximate value for the positioning RMSE in the room model space, the room floor was selected as a reference plane. Fig. 9 shows the positioning RMSE results using three source points and a point PN located at any location on the floor of the room. In the figure, the $\mathrm{X}$ and $\mathrm{Y}$ axes represent the length and width of the room, and the $\mathrm{Z}$ axis indicates the positioning RMSE result value corresponding to the ground position. The source coordinates used for this point were $(159,304,300),(455,455,300)$, and $(296,166,300)$. It can be seen that the positioning errors at different locations on the room floor differ, and that the RMSE results show a general centralization trend. The RMSE value is smaller and the positioning accuracy higher when PN approached the source location. Similarly, the RMSE values were larger and positioning accuracy lower when PN was far from the source location. 


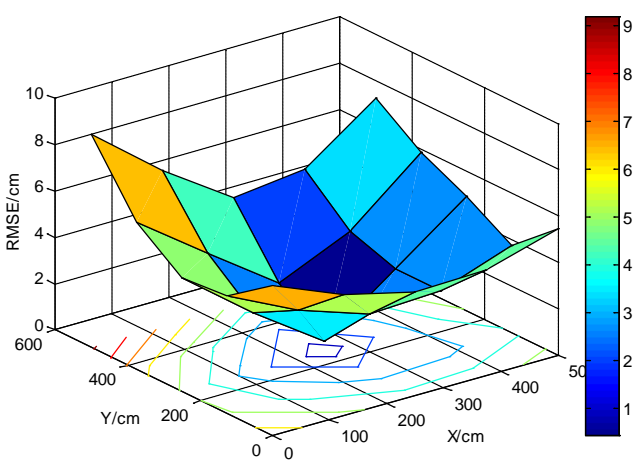

Fig. 9. RMSE distribution of PTCDV-Hop algorithm.

Fig. 10 compares the RMSE average value and number of source nodes for the purpose of PTCDV-Hop, TOA, DH-RLS, and traditional DV-hop algorithm. The line with symbol "diamond" represents the TOA algorithm, that with symbol "circle" represents the traditional DV-hop algorithm, that with symbol "asterisk" represents the DH-RLS algorithm, and that with symbol "upward-pointing triangle" represents the PTCDV-Hop algorithm. It can be seen that the average RMSE of the PTCDV-Hop algorithm is smallest, as the line with symbol "upward-pointing triangle" generally remains below other lines for the same source numbers. This supports the conclusion that the PTCDV-Hop algorithm has the best positioning accuracy among all algorithms. It should be noted that the downward trend in the graph shows a slight undulation because parameters such as the number of hops and average hop distance in the communication network are different at each position owing to the random determination of each source and PN during the simulation positioning process. Thus, it can additionally be determined that the average RMSE of the four positioning algorithms tend to decrease as the number of sources increase within a certain range.

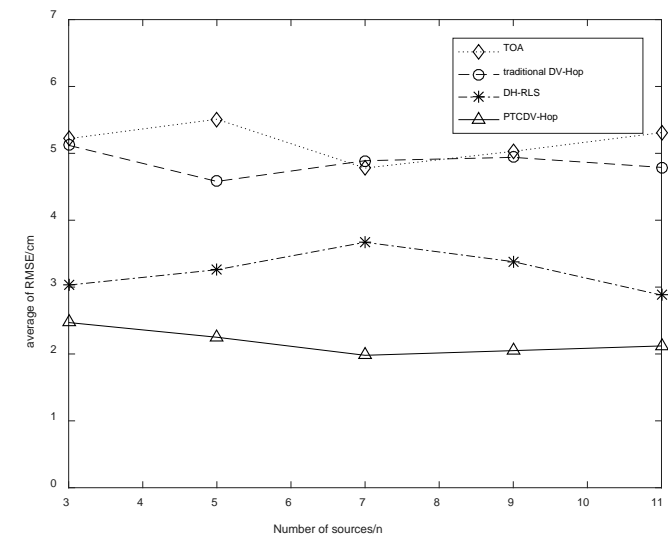

Fig. 10. Comparison of RMSE average value 


\section{Conclusion}

LED-based VLC technology has developed rapidly, becoming regarded as one of the most promising communication technologies of the future by combining communication with dayto-day lighting, thereby reducing the cost of communication equipment and benefitting the applicability. Compared to radio frequency communication, optical communication reduces electromagnetic radiation and electromagnetic interference, and is more conducive to longterm use. VLC systems may thus be deployed in indoor environments for communication and positioning. This paper proposed a PTCDV-hop location algorithm based on LED SAHP. The algorithm projects the distance between source nodes and a node to be positioned (located in a 3D indoor space) onto a 2D plane. Circles are defined by taking the source nodes (projected onto the horizontal plane of the node to be positioned) as the centers and the projection distances as the radii. The proposed algorithm then determines the location coordinates of the node to be positioned using the trilateral-weighted-centroid algorithm. Simulation results verified the rationality of the theoretical analysis and the advantages of the proposed algorithm.

\section{Reference}

[1] Bashiri, M., and M. Rezanezhad, "A reliable multi-objective p-hub covering location problem considering of hubs capabilities," International Journal of Engineering Transactions B Applications, vol. 28, no.5, pp. 717-729, January 29, 2015.

[2] M. Mohammadkhanloo and M. Bashiri, "A Clustering Based Location-allocation Problem Considering Transportation Costs and Statistical Properties," International Journal of Engineering Transactions B Applications, vol. 26, no. 6, pp. 597-604, January 24, 2013.

[3] Liang S, Zhang Y, Fan B, et al, "Multi-attribute Vertical Handover Decision-making Algorithm in a Hybrid VLC-Femto System," IEEE Communications Letters, vol. 21, no. 7, pp. 1521-1524, January 19, 2017. Article (CrossRef Link).

[4] Bo Fan, Hui Tian, Yuexia Zhang, Yuan Zhang, "A Virtual MAC Concept and its Protocol Design in Virtualized Heterogeneous Wireless Network," IET Communications, vol. 11, no. 1, pp. 53-60, December 22, 2016. Article (CrossRef Link).

[5] Lu Li, Yuexia Zhang, Bo Fan, Hui Tian, "Mobility-Aware Load Balancing Scheme in Hybrid VLC-LTE Networks," IEEE Communications Letters, vol.20, no.11, pp. 2276-2279, August 8, 2016. Article (CrossRef Link).

[6] Luo J, Fan L, Li H, "Indoor Positioning Systems Based on Visible Light Communication: State of the Art," IEEE Communications Surveys \& Tutorials, vol. 19, no. 4, pp. 2871-2893, August 23, 2017. Article (CrossRef Link).

[7] H. S. Kim, D. R. Kim, S. H. Yang, Y. H. Son and S. K. Han, "An Indoor Visible Light Communication Positioning System Using a RF Carrier Allocation Technique," Journal of Lightwave Technology, vol. 31, no. 1, pp. 134-144, January 1, 2013. Article (CrossRef Link).

[8] Do T H, Hwang J, Yoo M, "Enhanced VLC-TDoA Algorithm for Indoor Positioning Without LED-ID,” American Journal of Surgical Pathology, vol. 38, no. 8, pp. 672-678, August, 2013. Article (CrossRef Link).

[9] Nan W, Xudong W, Qingqing H, et al, "Multiple LED Based High Accuracy Indoor Visible Light Positioning Scheme," Journal of Electronics \& Information Technology, vol. 37, no. 3, pp. 727732, March, 2015. Article (CrossRef Link). 
[10] Xudong W, Qingqing H, Nan W, "High accuracy indoor visible light positioning algorithm," Journal of Optoelectronics• Laser, vol 26, no. 5, pp. 862-868, May, 2015.

Article (CrossRef Link).

[11] X. Zhang, J. Duan, Y. Fu and A. Shi, "Theoretical Accuracy Analysis of Indoor Visible Light Communication Positioning System Based on Received Signal Strength Indicator," Journal of Lightwave Technology, vol. 32, no. 21, pp. 4180-4186, November, 2014. Article (CrossRef Link).

[12] Wang P, He J, Xu L, et al, "Characteristic Modeling of TOA Ranging Error in Rotating AnchorBased Relative Positioning,” IEEE Sensors Journal, vol. 17, no. 23, pp. 7945-7953, September 28, 2017. Article (CrossRef Link).

[13] Li S, Hedley M, Collings I B, et al, "TDOA-Based Localization for Semi-Static Targets in NLOS Environments," IEEE Wireless Communications Letters, vol. 4, no.5, pp. 513-516, June 24, 2015. Article (CrossRef Link).

[14] Hou Y, Xiao S, Bi M, et al, "Single LED Beacon-Based 3-D Indoor Positioning Using Off-theShelf Devices,” IEEE Photonics Journal, vol. 8, no. 6, pp. 1-11, December 7, 2016.

Article (CrossRef Link).

[15] Xiaoyin Li, Lianshan Yan, Wei Pan, Bin Luo, "Secure and Robust DV-Hop Localization Based on the Vector Refinement Feedback Method for Wireless Sensor Networks," The Computer Journal, vol. 60, no. 6, pp. 810-821, June 1, 2017. Article (CrossRef Link).

[16] N. A. M. Maung, M. Kawai, "Experimental evaluations of RSS threshold-based optimised DVHOP localisation for wireless ad-hoc networks," Electronics Letters, vol. 50, no. 17, pp. 12461248, August 25, 2014. Article (CrossRef Link).

[17] Guan W, Wu Y, Wen S, et al, "A novel three-dimensional indoor positioning algorithm design based on visible light communication," Optics Communications, vol. 392, pp. 282-293, June 1, 2017. Article (CrossRef Link).

[18] Bassem Fahs, Jeffrey Chellis, et al, "A 6-m OOK VLC Link Using CMOS-Compatible p-n Photodiode and Red LED,” IEEE Photonics Technology Letters, vol. 28, no. 24, pp. 2846-2849, November 1, 2016. Article (CrossRef Link).

[19] Zunaira Babar, Mohd Azri Mohd Izhar, et al, "Unary-Coded Dimming Control Improves ONOFF Keying Visible Light Communication,” IEEE Transactions on Communications, vol. 66, no. 1, pp. 255-264, October 4, 2017. Article (CrossRef Link).

[20] M. Sönmez, "Simplified and accelerated PPM receivers for VLC systems," IET Optoelectronics, vol. 12, no. 1, pp. 36-43, January 15, 2018. Article (CrossRef Link).

[21] Jiankun Z, et al, "Modulation Scheme Analysis of Indoor Visible Light Communications," Chinese Journal of Lasers, Vol. 38, no. 4, pp. 131-134, April 2011. Article (CrossRef Link).

[22] Quan Sun, Weida Zhan, Zhuo Gao, Ziqiang Hao, "Differential Multi Pulse Position Amplitude Modulation in Optical Communications,” Boletín Técnico, vol. 55, no. 7, pp. 1-7, September 12, 2017.

[23] Simin Khazraei, Mohammad Amin Shoaie, Mohammad Reza Pakravan, "Efficient modulation technique for optical code division multiple access networks: differential pulse position modulation,” IET Optoelectronics, vol. 8, no. 5, pp. 181-190, September 8, 2014.

Article (CrossRef Link).

[24] Renbo Liao, Hongzhan Liu, Yaojun Qiao, "New hybrid reverse differential pulse position width modulation scheme for wireless optical communication," Optical Engineering, vol. 53, no. 5, pp. 1-8, May 21, 2014. Article (CrossRef Link).

[25] Qing Z, et al, "Unidirectional visible light communication system based on narrow band-pass amplifier," Journal of Optoelectronics•laser, vol. 28, no.1, pp. 38-43, January, 2017.

Article (CrossRef Link). 
[26] Li F, Wu K, Zou W, et al, "Analysis of energy saving ability in dimming VLC systems using LEDs with optimized SAHP,” Optics Communications, vol. 361, pp. 86-96, February 15, 2016. Article (CrossRef Link).

[27] Zhihua Cui, Yang Cao, Xingjuan Cai, Jianghui Cai, Jinjun Chen, "Optimal LEACH protocol with modified bat algorithm for big data sensing systems in Internet of Things," Journal of Parallel and Distributed Computing, vol. 132, pp. 217-229, 2019. Article (CrossRef Link).

[28] Guanhua Z, Ligang H, Jiangtao C, "Research on Centroid Localization Algorithm of Building 3D Projection Based on DV-hop Algorithm,” Measurement \&Control Technology, vol. 34, no. 2, pp. 118-124, February, 2015. Article (CrossRef Link).

[29]Zhihua Cui, Bin Sun, Gaige Wang, Yu Xue, Jinjun Chen, "A novel oriented cuckoo search algorithm to improve DV-Hop performance for cyber-physical systems," Journal of Parallel and Distributed Computing, vol. 103, pp. 42-52, May, 2017. Article (CrossRef Link).

[30] Farrukh Shahzad, Tarek R. Sheltami, Elhadi M. Shakshuki, "DV-maxHop: A Fast and Accurate Range-Free Localization Algorithm for Anisotropic Wireless Networks," IEEE Transactions on Mobile Computing, vol. 16, no. 9, pp. 2494-2505, November 24, 2016. Article (CrossRef Link).

[31] Lina Zhou, Guangjie Han, Li Liu, "Pulse-Based Distance Accumulation Localization Algorithm for Wireless Nanosensor Networks,” IEEE Access, vol. 5, pp. 14380-14390, July 27, 2017. Article (CrossRef Link).

[32] Bang Wang, Guang Wu, Shu Wang, "Localization Based on Adaptive Regulated Neighborhood Distance for Wireless Sensor Networks With a General Radio Propagation Model," IEEE Sensors Journal, vol.14, no. 11, pp. 3754-3762, May 14, 2014. Article (CrossRef Link).

[33] Yuexia Zhang, Hang Chen, "Research on IRT-N Triangle Array Based on LED SAHP," Semiconductor Optoelectronics, 2018. 


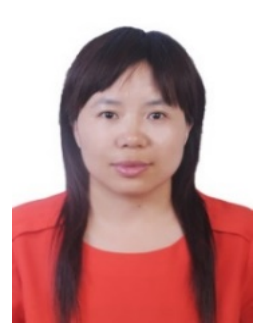

Yuexia Zhang was born in Anyang Village, Henan Province, China in 1978. She received the B.S. degree in detection technology and instrument from Zhengzhou University, Henan, in 2001. She received the M.S. degree and Ph.D. degree in information and communication engineering for Beijing University of Posts and Telecommunications Beijing, in 2008.

From 2008 to now, she was an Assistant Professor with the School of Information and Communication Engineering, Beijing Information Science and Technology University. She is the author of three books, more than 20 articles, and more than 10 inventions. Her research interests include wireless cooperative communication technology, ultra-wideband technology, and wireless positioning technology. She holds two patents.

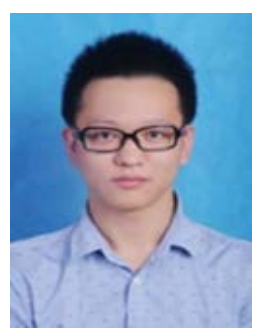

Hang Chen received the B.E in network engineering from Liaoning University of Technology in 2015. He has published papers about routing algorithms and LED half power angle visible light indoor positioning methods and other papers. He is mainly engaged in mobile communication and $5 \mathrm{G}$ positioning.

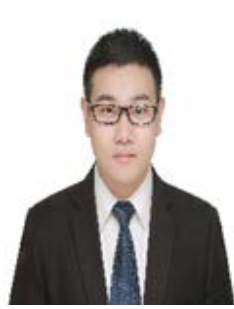

Jiacheng Jin received the B.E.E degrees in electrical engineering from Beijing Information Science and Technology University in 2017. At present, he is a graduate student of Beijing Information Science and Technology University. He is mainly engaged in visible light localization and 5G orientation research. 